\title{
Data hiding error concealment for JPEG2000 images
}

\author{
Milan Brezina, Kamil Bodecek, Milan Brezina, \\ Faculty of Electrical Engineering and Communication, \\ Brno University of Technology \\ Purkynova 118, 612 00 Brno, Czech Republic \\ imb@email.cz, kamil.bodecek@phd.feec.vutbr.cz, brezina.milan@email.cz
}

\begin{abstract}
This paper presents an error concealment technique for image transmission in JPEG2000 for the lowest frequency coefficients. The proposed method uses the layer structure that is a feature of the JPEG2000. The most significant layer is hidden in place of the lowest layer of the bit stream. After transmission this embedded layer is used for error concealment. The bit stream encoded using the proposed method has the same data structure as the standard JPEG2000. Experiments show the effectiveness of these algorithms.
\end{abstract}

Keywords: Error concealment, data hiding, JPEG2000, layer structure.

\section{Introduction}

Multimedia communication through wireless and broadcast network is becoming increasingly important. Since these networks transmit data with a very high bit error rate and packet losses, some regions of the transmitted data may not be decoded. Error resilience issue has become a necessity. Error concealment (EC) is an effective error resilience method which combats against the effects of residual errors staying in the bit stream after transmission.

The standard JPEG2000 includes some error resilience tools such as entropy coding, packet markers and also data partitioning. These tools are used to detect and locate possible errors, and also to resynchronize the decoding process. They can minimize the impact of errors in images, but are only applied at the source coding stage. The errors during transmission can still cause the loss of some wavelet coefficients [3]. Any EC method has not been standardized for JPEG2000 yet. Only JPEG2000 VM 7.2 proposes to replace the lost wavelet coefficients by zeros, which will affect the image quality in a certain extent. New efficient EC techniques for JPEG2000 are necessary. Some of the proposed EC methods for JPEG2000 actually aimed at the study of wavelet-based coding [2], [3], [4] and [5]. In a recent work [6], the authors proposed to use the data hiding to facilitate EC, which employed the layer structure of a JPEG2000 bit stream. The method required that the length of the least significant

Please use the following format when citing this chapter:

Brezina, M., Bodecek, K., Brezina, M., 2007, in IFIP International Federation for Information Processing, Volume 245 ,

Personal Wireless Communications, eds. Simak, B., Bestak, R., Kozowska, E., (Boston: Springer), pp. 505-513. 
layer must be the same as that of the most significant layer. Fig. 1 shows the case for 20 layers. Our proposed method uses linear bit distribution which causes that all layers in the bit stream have nearly same length as required in [6] as well. It means that each layer has the same quality increment. Kakadu software employed in this work uses logarithmical distribution in general which is not suitable and the distribution has to be add set up manually. The last geometrical distribution is shown as an example when the goal bit rate is divided by 2 in each step for layer. It means last significant layer includes $50 \%$ of quality increment, the following $25 \%$ and so on.

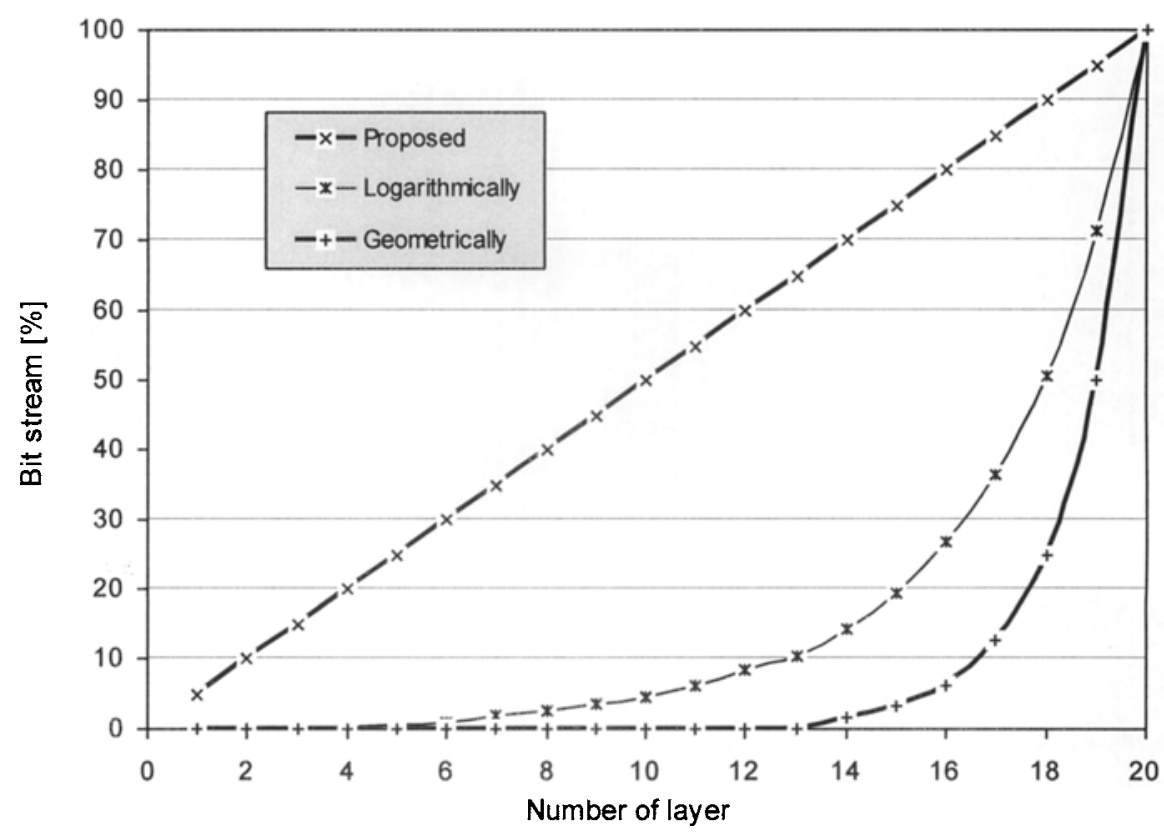

Fig. 1. Bit distribution in layers

This paper is organized as follows. Section 2 briefly reviews the features of the JPEG2000 encoder and the general concept of exploiting the data hiding to facilitate error concealment. Section 3 describes the proposed method in the details. Following experimental results are presented in Section 4. Section 5 is a conclusion of this paper.

\section{JPEG2000 encoder}

The JPEG2000 standard has a lot of useable features which were described in many previous papers of many authors. The most important of them are error resilient tools and scalabilities. Scalable coding means that image can be simultaneously available for decoding at a variety of qualities or resolutions. The lowest units of JPEG2000 
code stream is a packet. Packets contain bit data from a certain layer, component, resolution, and precinct of one tile (see Fig. 2). The order in which these packets are arranged is called as the progression order [2]. The standard defines four different progression orders. The most used is RLCP or LRCP. For example the Layer Resolution - Component - Position progression is defined as the interleaving of the packets in the following order:

$$
\begin{aligned}
& \text { for each } 1=0, \ldots, N-1 \\
& \text { for each } r=0, \ldots, R \\
& \text { for each } i=0, \ldots, I \\
& \text { for each } k=0, \ldots, K
\end{aligned}
$$

packet for layer $l$, resolution $r$, component $i$ and precinct $k$, where $N$ is number of layers, $R$ is maximum number of decomposition levels, $I$ is number of component and $K$ is number of precincts. Obviously by encoding, the number of components and precincts are equal number one.

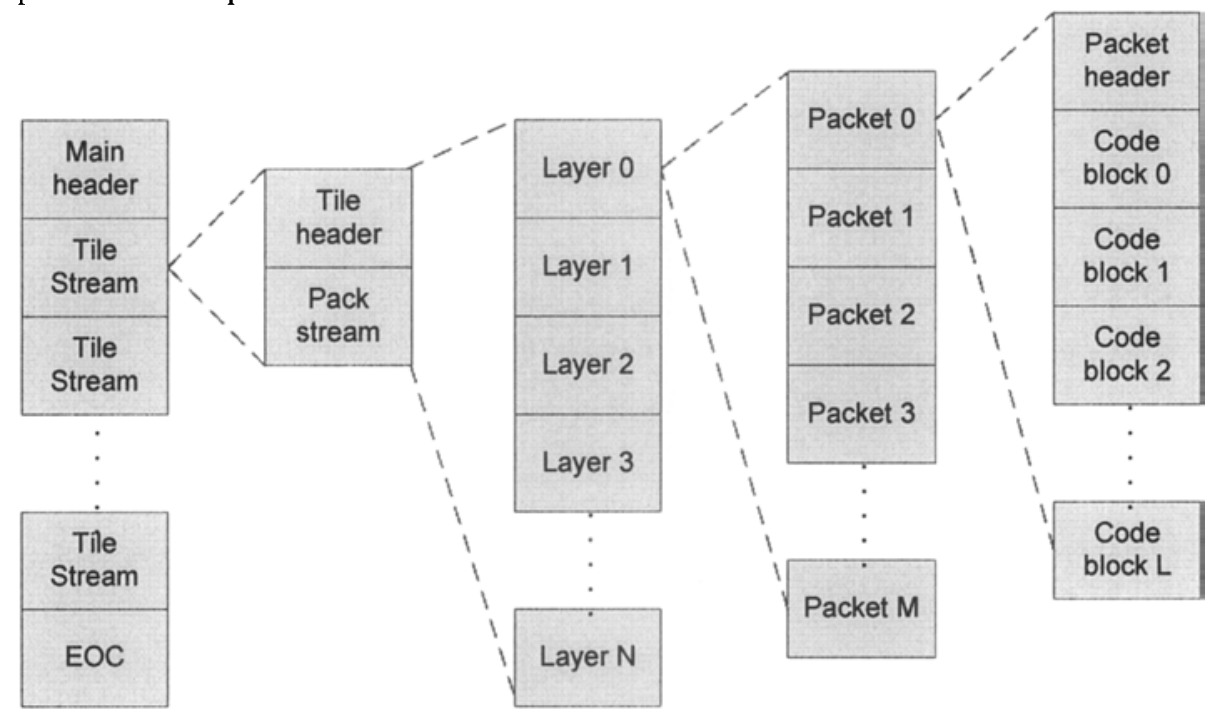

Fig. 2. JPEG2000 code stream structure

In addition, the JPEG2000 decoder can generate an image with a lower bit rate by using the upper layers or by truncating the lower layers of the bit stream. It means that an image with an arbitrary bit rate can be decoded from one JPEG2000 bit stream in the decoder side. It should be noted that the number of layers and the length of each layer can be assigned arbitrarily in the JPEG2000 encoding process [6]. Note the number of generated packets is expressed as a multiplication of the number of layers by the number of resolution levels. Since the size of a packet is determined automatically, we cannot assign an arbitrary size. 
As it was mentioned above the JPEG2000 standard has error resilience tools which combat against destructive impact of transmission errors. Error resilience is achieved at the entropy coding and packet level. At the entropy coding level, to localize random and burst errors, the arithmetic coder can be terminated after every coding pass. This allows the decoder to continue the decoding process if errors are detected. At the packet level, the situation is very similar. The decoder can detect and localize errors in the packets. In case of data loss, the result cannot be achieved by using standard error resilience tools. In particular, when the significant layer or layers are lost, there is significant image distortion.

\section{Proposed method}

First at all a layer structure has to be formed for scalability of a bit stream to use this method. Figure 3 shows a block diagram of a JPEG2000 extended coder which hides the highest layer in the lowest layer. Each layer with a specific data size is generated using the standard JPEG2000 encoder. After that the least significant layer is replaced by the most significant layer (see Figure 4).

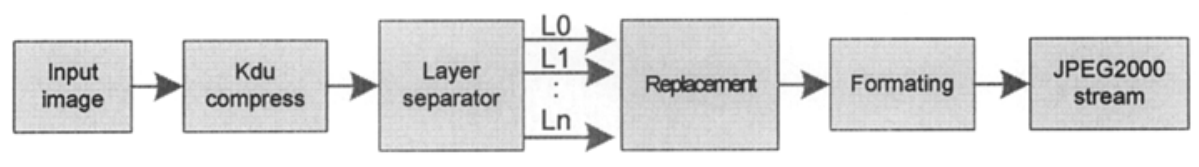

Fig. 3.Block diagram of extended JPEG2000 coder

Original code stream

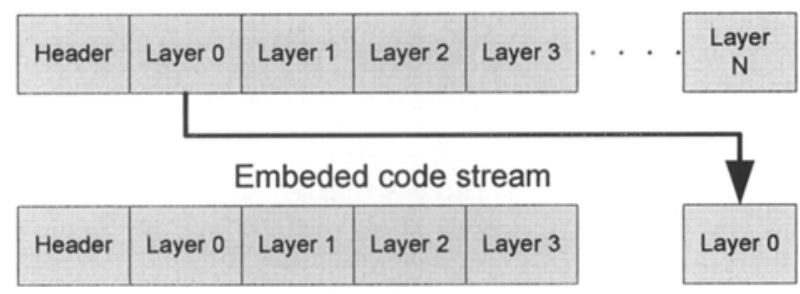

Fig. 4.Proposed encoder extension

Generally it works as follows; the most significant layer (Layer 0) is duplicated. One of duplicates is placed instead of the highest layer second one is placed as a first layer in the bit stream. The length of the least and the most significant layers has to be the same to do mentioned process. The length of the bit stream with the embedded data is therefore the same as that of the original file. If the encoded bit stream with embedded data has the same structure as the original stream, a standard decoder tools can decode this stream. In addition we can use also the JPEG2000 error resilience tools. Finally the least significant layer to inhibit the image deterioration caused by the data hiding, that data is set to zero [7]. 
The errors mainly are burst errors in the general wireless channel or in Internet, so the probability that the error position of the first packets is the same as that of the hidden data is very small. The reason why we duplicate the most significant layer is that the most important data (low wavelet coefficients) are included in the most significant layer, in our case it is layer 0 . Wavelet coefficients are separated into the bit-planes from MSB to LSB. In this way if the upper bit-plane coefficients included in the upper significant layer are affected by errors, the other coefficients of lower bitplane(s) are getting to be meaningless. Therefore error concealment of these coefficients is desired. Figure 5 shows how the mentioned method reduces the goal PSNR of much known testing pictures in comparison to the images with only the most significant layer 0 . You can see differences between using Layer 0 and Layer Max in particular pictures. But differences between picture with all layers (Layer Max) and pictures with hiding data (Max -1 ) is varying from $0.1 \mathrm{~dB}$ to $0.3 \mathrm{~dB}$.

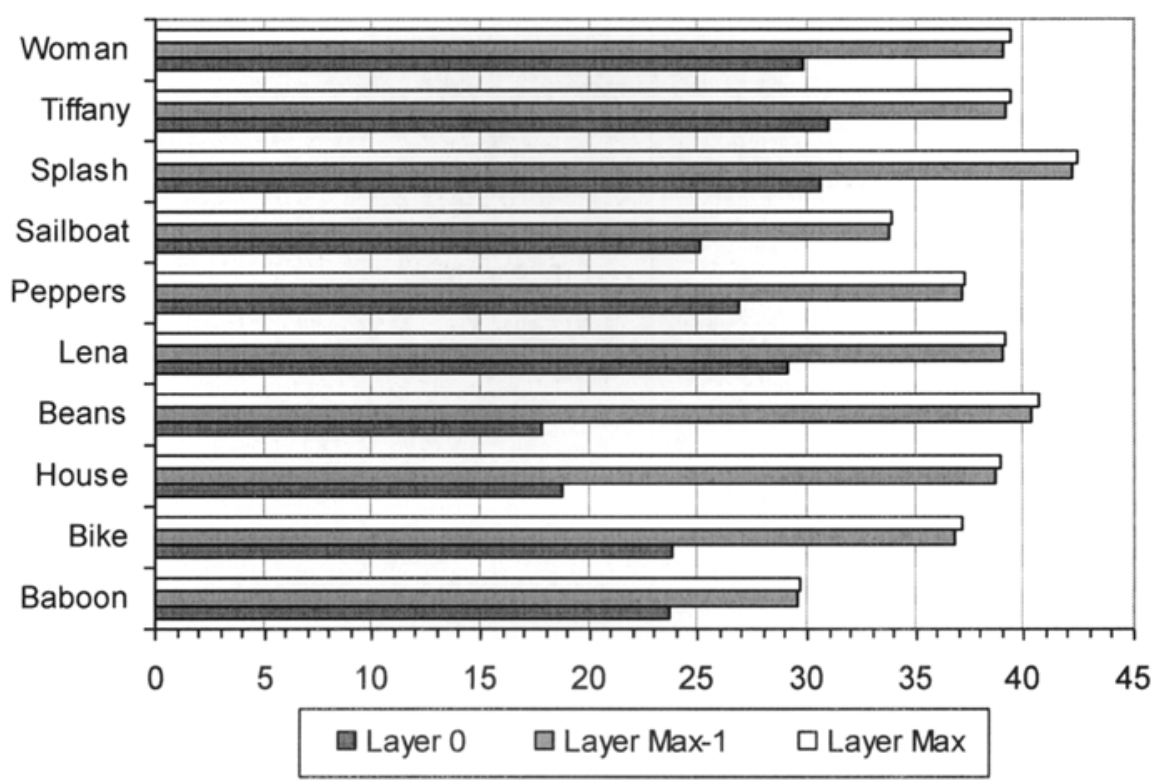

Fig. 5. PSNR of test pictures for $1 \mathrm{bpp}$

Figures 6 and 7 show a block diagram of an extended JPEG2000 decoder and error concealment technique. In the concealment process, errors are concealed using embedded data. The embedded layer is compared with the most significant layer. When this layer is affected by errors, the data of this layer are concealed by the hidden data of embedded layer. There are two main types of problems; random errors and packet losses. Random errors can be localized in each code-block and coding pass. Therefore these errors are concealed using the code-block and pass data of hidden layer. On the other side packet losses are detected using packet header and resynchronization symbols. Packet losses from the most significant layer are replaced by packets extracted from the hidden layer. 


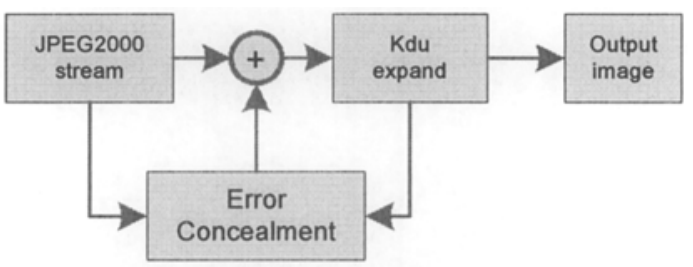

Fig. 6.Block diagram of extended JPEG2000 decoder

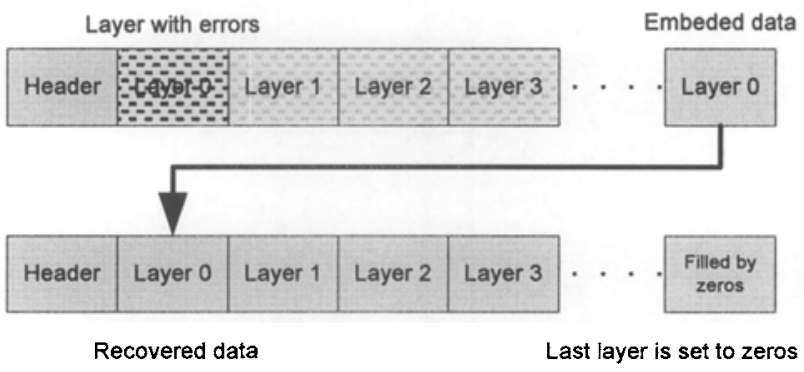

Fig. 7. Proposed error concealment process

\section{Experimental results}

We use simulations to establish the performance of the proposed method. The method was applied to a 10 standard black and white and color images in original resolution. Image coding was done using 5-level composition process with reversible wavelet filters. The target bit rate was $0.5 \mathrm{bit} / \mathrm{pixel}$. A bit stream was consisted of 10 layers. Note the most significant layer is layer 0 . Image quality was measured in terms of the peak signal to noise ratio (PSNR) between the original and the decoded image.

\subsection{Images without errors}

The image distortion caused by hiding layer 0 in layer 9 of a bit stream is shown here. The original compressed image without hidden data is in Figure $8 \mathrm{a}$ and its PSNR is $32.8 \mathrm{~dB}$. Figure $8 \mathrm{~b}$ shows image with the only layer 0 and its PSNR is $23.9 \mathrm{~dB}$. The last image in Figure $8 \mathrm{c}$ shows image with hiding data in the least significant layer 9 with PSNR $32.6 \mathrm{~dB}$. Additionally the hidden data is truncated from the bit stream during the decoding process. As these figure showed, the proposed method can hide certain amount of data without apparent deterioration. Embedded layer makes this deterioration about $0.2 \mathrm{~dB}$. This means that the image deterioration caused by hiding data can be easily ignored. Using more number of layers and higher bit rate the embedded deterioration is going to be meaningless. 


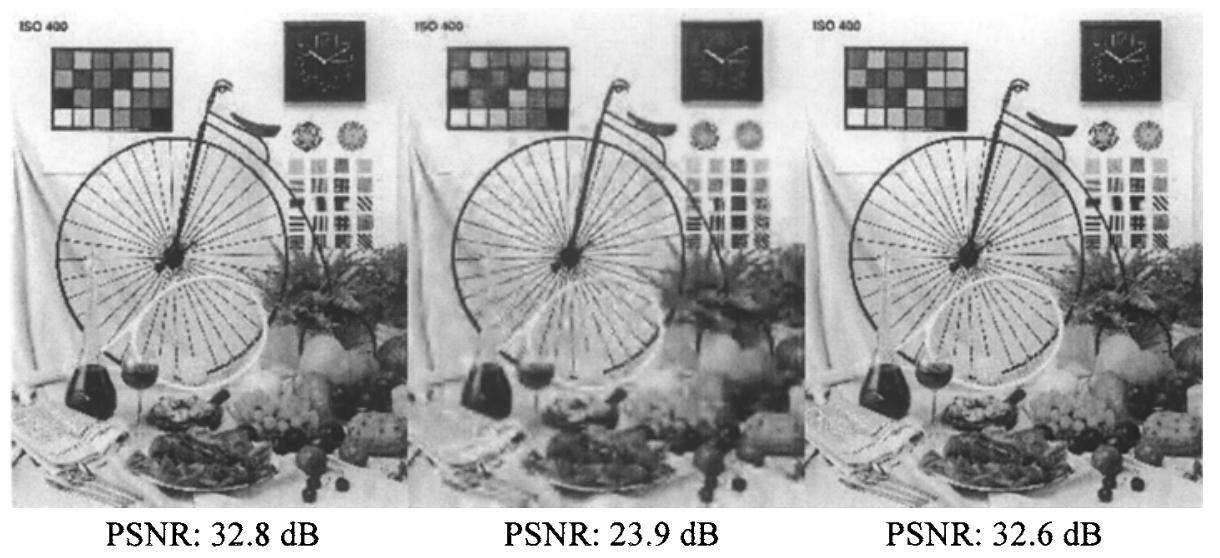

Fig. 8. Visual quality of JPEG2000 images (a) original, (b) only Layer 0, (c) Layer N-1

\subsection{Images with errors}

In this section we can compare the quality of the proposed error concealment in relation to burst errors. It is assumed that errors are detected perfectly by using error resilient tools. The errors are generated randomly and independently at a rate of $10^{-3}$. Figures 9 and 10 show images decoded from erroneous stream without and with using the JPEG2000 error resilient tools, respectively. Right parts of figures show the concealed images decoded by the proposed method. These figures show that the proposed method improves the visual image quality as well as its PSNR. Moreover, using JPEG2000 error resilient tools, we obtain better goal image quality after using error concealment even the PSNR difference between image with and without using error concealment is little bit smaller than without using these tools [8], [9], [10].

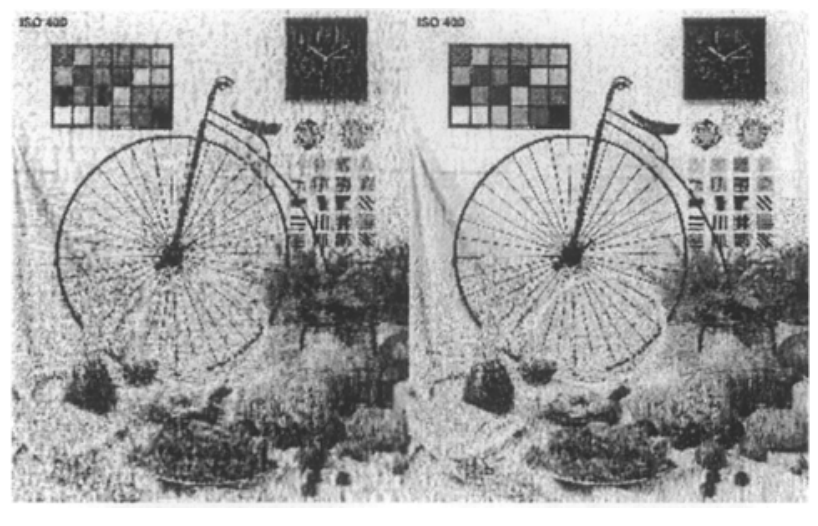

PSNR: $16.17 \mathrm{~dB}$

PSNR: $21.71 \mathrm{~dB}$

Fig. 9. Images decoded without JPEG2000 error resilient tools 


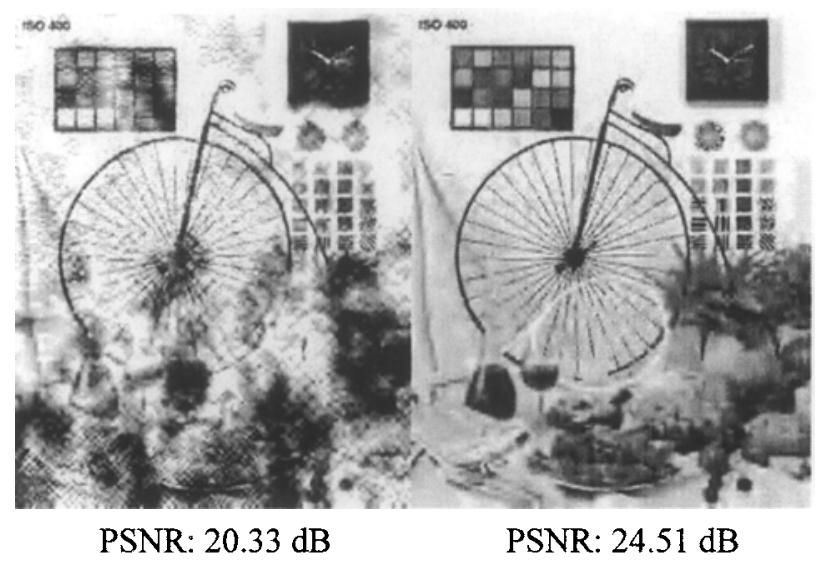

Fig. 10. Images decoded with JPEG2000 error resilient tools

Note that it is best to do this kind of experiments on larger images. Obviously used only Lena $(512 \times 512)$ image is extremely old. Modern image compression applications more often than not involve images with at least a few mage-pixels. Lena and the other USC images were scanned from magazines in the $1980^{\prime} \mathrm{s}$ using a scanner whose color channels were not even properly aligned.

\section{Conclusion}

We have presented an efficient error concealment method which is proposed aiming at the recovery of the missing visual information in the transmission of JPEG2000 images over error prone channels. This method is based on the features of JPEG2000 standard at the layer level of a bit stream. Error concealment is implemented by adopting the data hiding technique.

The proposed method is available for any error correcting codes. Thus, under a higher error rate a more powerful code can be used for the error concealment. Moreover it should be noted that a bit stream produced by the proposed method with using a code has the same data structure as a standard JPEG2000. Simulation results showed that hiding data had only a small effect on image quality in comparison with the quality of the proposed error concealment method.

\section{Acknowledgment}

This article was created with aid of project FRVŠ 1363/2007. 


\section{References}

1. ISO/IEC 15444-1, JPEG2000 Part 1 final draft international standard, Aug. 2000.

2. Taubman, D., Marcelin, M. W.: JPEG2000 Image compression fundamentals, standard and practice, Kluwer academic publisher (2001)

3. Moccagatta, I., Soudagar, S., Liang, J., Chen, H.: Errorresilient coding in JPEG2000 and MPEG-4, IEEE Journal on Selected Areas in Communications, (2000) vol. 18 , pp. 899-914

4. Shirani, S., Kossentini, F., Ward, R.: Error concealment methods: A comparative study, In IEEE Canadian Konference on electrical and computer engineering, Edmonton, Canada, (1999) pp. 835-840

5. Atzori, L., Dodona, S., Giusto, D., D.: Error recovery in JPEG2000 image transmission, in Proc. IEEE ICASSP, Salt Lake City, USA, (2001) vol. 3, pp. 1733-1736

6. Kurosaki, M., Kiya, H., Error concealment using layer structure for JPEG2000 images, in Proc. 15th Workshop on Circuits and Systems in Karuizawa of IEICE, Japanese, pp.77-82, (2002)

7. Lee, P., G., Chen, L., G.: Bit-plane error recovery via Gross subband for image transmission in JPEG2000, IEEE International Conference on Multimedia and Expo, Lausanne pp. 149-152, (2002).

8. Brezina, M.,: Transmission of still images JPEG2000, Telecommunication and Signal Processing, Brno: Brno University of Technology (2005)

9. Brezina, M.,: JPEG2000 transmission over lossy channel, Research in Telecommunication Technology, Brno: Brno University of Technology (2005)

10. Březina, M.: Wavelet coding for mobile applications, Research in Telecommunication Technology, Brno: Brno University of Technology (2005) 